\title{
Cardiovascular Complications in Diabetes
}

\author{
Roever L*, Casella-Filho A, Dourado PMM, Resende ES and Chagas ACP
}

Heart Institute (InCor), HCFMUSP- University of São Paulo Medical School, São Paulo, Brazil, Federal University of Uberlândia and Faculty of Medicine ABC, Santo André, Brazil

\begin{abstract}
Cardiovascular disease is the primary cause of morbidity and mortality among the diabetic population. Diabetic cardiomyopathy is characterized by early impairments in diastolic function, accompanied by the development of cardiomyocyte hypertrophy, myocardial fibrosis and cardiomyocyte apoptosis. The pathophysiology underlying diabetes-induced cardiac damage is complex and multifactorial, with elevated oxidative stress as a key contributor. The current evidence of molecular disturbances present in the diabetic heart, and their role in the development of diabetes-induced impairments in myocardial function and structure. This review aims to address the recent aspects of the relationships between diabetic cardiomyopathy and cardiovascular disease.
\end{abstract}

Keywords: Diabetic cardiomyopathy; Risk factors; Myocardial function; Cardiomyocyte; Cardiovascular disease

\section{Introduction}

Morbidity and mortality among people with diabetes mellitus are mostly triggered by premature Cardiovascular Disease (CVD) $[1,2]$. An estimated 285 million adults globally were burdened by this chronic disease in 2010; this number is projected to increase to 439 million by 2030 [3]. At least 10.3 million Americans carry a diagnosis of diabetes mellitus and 5.4 million are estimated to have undiagnosed diabetes. Approximately $90 \%$ of patients with diabetes have the type 2 variety [3]. There is a lack of consensus regarding the pathogenesis and diagnosis of DC, and a standard treatment has yet to be established. Factors that are recognized to be involved in the pathogenesis of DC include metabolic disorders, myocardial fibrosis, microvascular disease, autonomic disorders and Insulin Resistance (IR) [4]. DC is a complex condition mediated by tissue specific and diabetes-related interconnected pathological processes. Focal cardiac fibrosis, a prominent cause for diabetic cardiomyopathy, is considered to be an early event, which sets the stage for heart failure by reducing contractile efficiency and demanding greater cardiac contractile force, eventually leading to cellular death $[5,6]$. Irrespective of the inciting event, several neurohormonal and inflammatory pathways are activated, including the Renin-Angiotensin- Aldosterone System (RAAS), adrenergic system, inflammatory cytokine and a host of other autocrine and paracrine mechanisms as compensatory mechanisms to maintain stroke volume at a reduced ejection fraction [6]. It is widely accepted that the diabetic heart is associated with Left Ventricular (LV) diastolic (and often systolic) dysfunction, cardiomyocyte hypertrophy, myocardial interstitial fibrosis, increased apoptosis and upregulation of oxidative stress, the pathophysiology of diabetic cardiomyopathy is thus complex and multifactorial [79]. The substancial number of studies have shown that diabetic cardiomyopathy is a source of functional and biochemical alterations (Figure 1). This review explores the current understanding of diabetic cardiomyopathy, and existing research supporting an association between diabetes and cardiovascular disease.

\section{Literature research}

A PubMed, Europubmed search was performed until April 2014 using the terms "Diabetic Cardiovascular Complications" in combination with the terms "micro-vascular", "macrovascular", "endothelium", or "cardiovascular".

\section{Diastolic dysfunction}

LV diastolic dysfunction is one of the first signs of diabetic cardiomyopathy, often developing before systolic dysfunction [8]. Diastolic dysfunction is characterized by symptoms of heart failure with preserved ejection fraction (usually equal to or greater than 0.45 ). Diastolic dysfunction is an integral characteristic of Heart Failure With Preserved Ejection Fraction (HFPEF) or diastolic heart failure, in which other impairments such as concentric hypertrophy and vascular stiffness are likely to also manifest [10]. Systolic and diastolic dysfunction can result in differences in gross cardiac morphology, associated for example with a dilated ventricle versus an absence of chamber dilation, respectively [11]. LV diastolic dysfunction, characterized by impaired and prolonged Iso volumetric Ventricular Relaxation Time (IVRT), can be reliably detected using imaging techniques such as Doppler echocardiography, tissue Doppler and magnetic resonance imaging $[12,13]$. Measures peak blood flow velocity across the mitral valve, to evaluate regional assessment of myocardial filling, using the ratio of the initial peak (E, early) and late (A, or atrial) blood flow velocity across the mitral valve. A reduced E/A ratio coupled with prolonged IVRT, indicative of diastolic dysfunction, are commonly observed in diabetic patients [14]. Diastolic early (E', to distinguish it from conventional transmitral $\mathrm{E}$ flow) and late ( $\mathrm{A}^{\prime}$, as distinct from conventional transmitral A flow) myocardial tissue velocities are derived by integrating these distances over time. LV filling pressure can also be estimated from tissue Doppler imaging, as the ratio of conventional early (E) transmitral flow velocity to diastolic early (E') tissue velocity (E/E') [13]. Cardiac magnetic resonance imaging has now emerged as another non-invasive technique for the measurement of cardiac function by providing a 3-dimensional representation of the structure of the heart. This technique yields the same indices of diastolic

*Corresponding author: Leonardo S Roever-Borges, MHS, Laboratory of Vascular Biology, Heart Institute (InCor), University of São Paulo Medical School, Av. Dr. Enéas de Carvalho Aguiar, 44 05403-900 São Paulo Brazil, Tel: 55-11 30695259; Fax: 55-11 30695261; E-mail: leonardroever@hotmail.com

Received May 10, 2014; Accepted August 12, 2014; Published August 20, 2014

Citation: Roever L, Casella-Filho A, Dourado PMM, Resende ES, Chagas ACP (2014) Cardiovascular Complications in Diabetes. J Diabetes Metab 5: 415 doi:10.4172/2155-6156.1000415

Copyright: @ 2014 Roever L, et al. This is an open-access article distributed under the terms of the Creative Commons Attribution License, which permits unrestricted use, distribution, and reproduction in any medium, provided the original author and source are credited. 


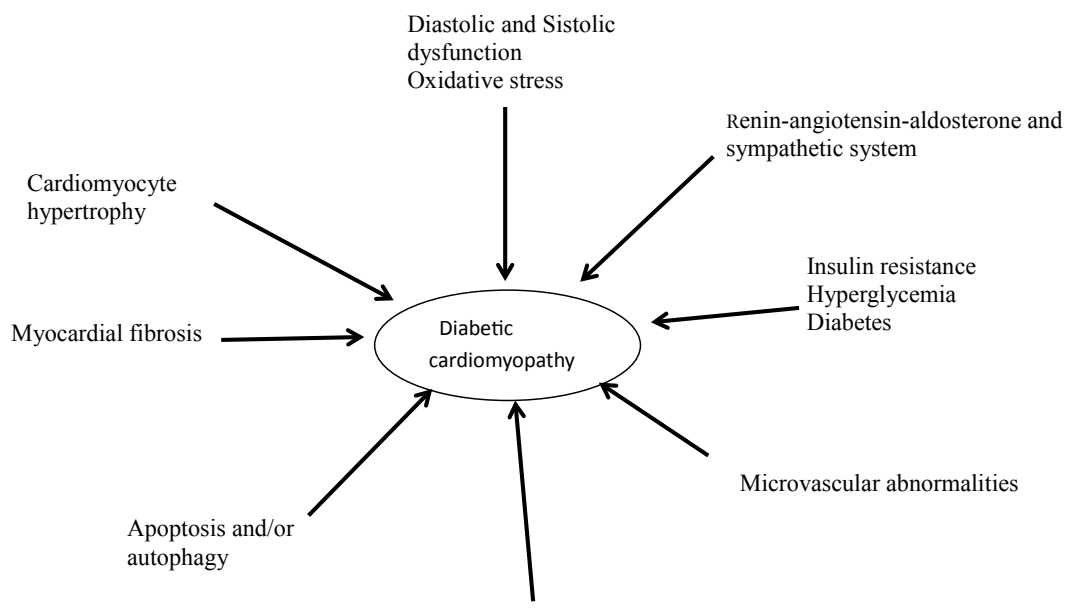

Impaired calcium homeostasis and dysfunction of mitochondria and endoplasmatic reticulum

Figure 1: Diabetic cardiomyopathy is a source of functional and biochemical alterations.

function as Doppler echocardiography (E/A, deceleration time, LV filling pressures), but with increased sensitivity and reproducibility. In asymptomatic patients with T2DM, cardiac magnetic resonance detected impairments in diastolic function, including reductions in reduced LV end-diastolic volume, $\mathrm{E}$ and $\mathrm{E} / \mathrm{A}$ [15]. Conventional echocardiography-derived measures of diastolic function such as E/A are heavily dependent on preload, load-independent measures such as $\mathrm{E} / \mathrm{E}$ ' derived from tissue Doppler more reliably describe the status of diastolic dysfunction, particularly in the latter stages of the disease [16]. Isolated LV diastolic dysfunction likely represents its initial stage of progression, although the mechanisms underlying this remain to be fully resolved. Likely contributors include insulin resistance, increased cardiac collagen deposition, changes in both glucose metabolism, generation of Reactive Oxygen Species (ROS) and Ca2+ homeostasis [17-19]. A substantial number of studies have shown that Perivascular Adipose Tissue (PVAT) is a source of predominantly pro-inflammatory, cytokines and chemokines (Figure 1).

\section{Systolic dysfunction}

The left ventricular systolic impairment is responsible for most cases of chronic heart failure and can be diagnosed by echocardiographic left ventricular ejection fraction less than or equal to 0.40 .

The majority of evidence regarding diabetic cardiomyopathy considers LV diastolic dysfunction to largely occur in isolation, or prior to LV systolic dysfunction, as outlined above. A smaller number of studies have however suggested that abnormal systolic function may also be evident, in both clinical and experimental settings [19]. Subclinical systolic dysfunction have been demonstrated in patients with T2DM, who exhibit regional impairments in LV systolic function, on both longitudinal and radial systolic velocities despite normal ejection fraction, peak systolic velocity and functional reserve [20].

\section{Cardiomyocyte hypertrophy}

The functional changes evident in the diabetic heart are closely associated with molecular and histopathological evidence of both cardiomyocyte hypertrophy and fibrosis. LV hypertrophy is a common structural hallmark in patients with diabetes, and is of clinical significance as it is a strong predictor of Myocardial Infarction (MI), stroke and death from HF [21]. The development of LV hypertrophy initially occurs as an adaptive response to elevated hemodynamic stress, reduced numbers of functional contractile cardiomyocytes and neurohormonal activation. Although LV hypertrophy is frequently associated with increased afterload in diabetic patients with hypertension, it can also occur independent of pressure-overload. Echocardiographic evidence revealed increased LV posterior wall thickness and mass, in addition to a greater ratio of wall thickness to chamber radius in diabetic patients, even in the absence of coronary artery disease or hypertension [22].

\section{Myocardial fibrosis}

The diabetic myocardium is the development of interstitial and/ or perivascular fibrosis. Extracellular matrix (ECM) is composed of collagen, elastin, laminin and fibronectin, which normally provide a scaffold for cardiomyocytes. Collagen is an integral component of the ECM, as it facilitates connections between cells and muscle bundles, maintaining myocardial structure, shape and chamber thickness [23]. Myocardial fibrosis is a result of abnormally elevated ECM deposition, in particular collagen, which increases myocardial stiffness. This impairs LV relaxation, with subsequent compromise in the efficiency of LV contraction. In the diabetic setting, a decrease in the activity of ECM degrading enzymes, Matrix Metalloproteinases (MMP), coupled with an increase in activity of their tissue inhibitors (TIMP) have been proposed as a mechanism underlying ECM accumulation [24,25]. Diabetes increases the proportion of collagen that is in the insoluble form, elevated myocardial content and gene expression of ECM proteins is often observed in experimental models of both T1DM and T2DM and is closely associated with impairments in LV diastolic filling [26,27]. Collagen type I and III are increased in the epicardial and perivascular regions of the human diabetic heart, whereas the endocardium accumulated collagen type IV. Diabetes-induced cardiac fibrosis is accompanied (and likely triggered) by the upregulation of transforming growth factor (TGF- $\beta 1$ ), its receptor TGF- $\beta$ receptor II, and its downstream mediator, Connective Tissue Growth Factor (CTGF) [28]. Alterations in glucose metabolism are an important contributing mechanism to diabetes induced cardiac remodeling. 
Hyperglycemia per se is sufficient to directly increase cardiac fibroblast and vascular smoothmuscle cell proliferation and elevating pro-growth signaling in cultured cardiomyocytes [29].

\section{Apoptosis and/or autophagy}

Apoptosis is the most well-known form of programmed cell death; tightly-controlled regulation of apoptosis is essential for maintaining tissue homeostasis under normal physiology [30]. Increased myocyte apoptosis is involved in the process of transition from the compensated to decompensated hypertrophic state in the diabetic heart, cardiomyocyte apoptosis is correlated with blood glucose levels [31]. Diabetesinduced cardiomyocyte apoptosis often occurs concomitantly with other structural anomalies including increased interstitial fibrosis and myofiber disarray, and is likely to be a direct result of hyperglycemiatriggered caspase- 3 activation $[32,33]$. Common markers of autophagy include microtubule-associated protein Light Chain 3 (LC3), double membrane vesicles on electron microscopy, autophagic flux, the ratio of the active LC3-II isoform to the inactive LC3-I isoform, as well as gene expression or protein levels of autophagy-related genes Atg5, Atg7, Beclin-1 and the LC3-binding protein p62. Autophagy is beneficial or detrimental in the heart remains controversial at present, with conflicting views surrounding excessive versus insufficient autophagy in the pathophysiology of cardiac dysfunction and cardiomyocyte death $[34,35]$. Altered regulation of various components of autophagic signaling in the diabetic heart may thus be a compensatory response to protect cells under conditions of cardiac stress. The contribution of this altered autophagic response to the pathogenesis of diabetic cardiomyopathy however requires further elucidation.

\section{Microvascular abnormalities}

Diabetic cardiomyopathy can develop independent of the macrovascular complications of the disease, structural and functional changes at the level of the coronary vasculature are a common comorbidity in diabetic patients, which can further aggravate diabetic cardiomyopathy. Sustained hyperglycemia is associated with endothelial dysfunction and the risk of enhanced microvascular permeability, impaired microvascular blood flow and subsequent tissue ischemia is increased [36,37]. Therapeutic approaches targeting impairments at the level of the coronary microvasculature may thus offer favorable benefits in the setting of diabetic cardiomyopathy.

\section{Hyperglycemia and hypoglycemia}

Aberrations in glucose control itself is sufficient to trigger an array of maladaptive processes including hyperinsulinemia and insulin resistance, Glucose Transporter-4 (GLUT-4) depletion, changes in Free Fatty Acid (FFA) oxidation, accumulation of Advanced Glycation End Products (AGEs), altered $\mathrm{Ca}^{2+}$ handing, increased generation of ROS and activation of the renin-angiotensin-aldosterone system (RAAS) [38]. Clinical studies have demonstrated a clear correlation between glycemic control and the risk of HF development and associated events, on both Fasting Plasma Glucose (FPG) and glycated hemoglobin (HbA1c) levels [39]. Uncontrolled hyperglycemia in T2DM patients without overt cardiomyopathy is associated with diastolic LV dysfunction, independent of obesity, dyslipidemia and systemic arterial hypertension [40]. Together, these studies emphasize the importance of effective glycemic control in the prevention of cardiovascular events in diabetic patients. The hemodynamic changes associated with hypoglycemia include an increase in heart rate and peripheral systolic blood pressure, a fall in central blood pressure, reduced peripheral arterial resistance, and increased myocardial contractility, stroke volume, and cardiac output.

\section{Insulin resistance}

Insulin resistance and the concomitant hyperinsulinemia, are significant risk factors for the development and progression of cardiovascular disease, exists to indicate a causal relationship between hyperinsulinemia, hypertension, metabolic syndrome and coronary artery disease [41].

Activation of the sympathetic nervous system one mechanism considered to underlie the development of high blood pressure under insulin-resistant settings, renal sodium retention and increased proliferation of vascular smooth muscle cells may also contribute [42]. Hyperinsulinemia is positively correlated with the risk of developing diabetes and coronary artery disease, as demonstrated by a number of studies [43]. The incidence of coronary artery disease associated with high triglycerides and low high-density lipoprotein levels is only significantly increased when accompanied by insulin resistance, even in the absence of diabetes [43]. Cardiac abnormalities, including LV hypertrophy, fibrosis and cardiomyocyte dysfunction are often already apparent in the prediabetic, insulin-resistant stage, as observed in animal models in vivo. In the diabetic heart, diminished activities of GLUT-4 results in reduced glucose utilization and impaired insulin signaling. This subsequently increases energy demand from FFA oxidation, raising myocardial oxygen demand and reducing cardiac efficiency, accompanied by dyslipidemia and lipotoxicity [44]. Cardiac insulin resistance include mitochondrial dysfunction, inflammation, cytokine upregulation, endoplasmic reticulum stress and stress kinase signaling, and metabolic derangements and insulin resistance precede the development of cardiac dysfunction and remodeling, these likely predispose the diabetic heart to damage [45-47].

\section{Diabetes}

At the cellular level, mitochondrial dysfunction in particular plays a significant contribution to the development and progression of both cardiac and vascular complications of diabetes. Alterations in mitochondrial morphology, (un)coupling, fission-fusion dynamics, $\mathrm{Ca}^{2+}$ load, substrate utilization and ATP generation are clearly both evident in, and exerting detrimental effects on the function of, the diabetic myocardium. Mitochondria also contribute as a pathophysiological trigger of diabetic cardiomyopathy as a key source of ROS in the heart [46-48]. Hypertriglyceridemia is a common feature of T2DM, characterized by decreased clearance of triglyceride-rich lipoprotein, due to a reduction in the levels of lipoprotein lipase or alterations in circulating lipoproteins [49]. Elevated triglyceride levels correlate with the severity of atherosclerosis and coronary heart disease in diabetic patients [50]. FFA levels are also elevated in T2DM, which may shed light on the mechanistic relationship between increased fat, insulin resistance, impaired glucose tolerance and central obesity [49]. FFA levels are elevated in pre-diabetic patients with impaired glucose tolerance, and both acute and chronic increases in FFA levels are sufficient to induce insulin resistance [51]. High circulating and cellular FFAs can directly elevate peripheral insulin resistance, stimulate apoptosis and trigger a harmful build-up of toxic intermediates which result in lipotoxicity and these deleterious effects can contribute to impaired cardiac function and adverse remodeling in the diabetic myocardium [52]. Reduced glucose uptake and metabolism postischemia may compromise the capacity of the diabetic heart to recover, and demonstrate greater LV dysfunction and severe structural remodeling in diabetic animals following short-term occlusion of the coronary artery [52]. 


\section{Neurohormonal activation}

The neurohormonal plasma profile of diabetic patients with HF is however generally similar to non-diabetic HF patients, with the exception of BNP, where plasma levels are further elevated in diabetic HF patients, perhaps as a result of diastolic dysfunction for which BNP has been considered as a prognostic marker [53,54]. Evident in the diabetic heart includes upregulation of the RAAS, ET- 1 and sympathetic nervous system, circulating Ang II, ET-1, the natriuretic peptides ANP and BNP, as well as catecholamines (epinephrine and norepinephrine) are also elevated [55].

\section{Oxidative stress}

Hyperglycaemia, a constant clinical sign of DM, has been shown to induce oxidative stress by stimulating the release of superoxide from mitochondria. This oxidant in turn causes DNA lesion and may lead to cell and tissue apoptosis [56]. It has been shown that oxidative stress can induce a cascade of apoptotic pathways. Studies conducted by Cai et al clearly showed that hyperglycaemia induced the release of reactive oxygen species, which stimulates cytochrome c-activated caspase-3 pathway [57]. Oxidative stress has been shown to significantly deplete the tissue concentration of apotransferrin, a crucial endogenous antioxidant. A reduced body content of apotransferrin is associated with an impaired antioxidant activity leading to enhanced lipid peroxidation [58]. One study, it was reported that the level of 4-hydroxy-2-nonenal, a by-product of lipid peroxidation, which inhibits glucose uptake into cells, is significantly elevated in the brain of diabetic animals and these factors may play a role in diabetes-induced myocardium damage that may result in HF $[59,60]$.

\section{Impaired calcium homeostasis and dysfunction of mitochondria and endoplasmatic reticulum}

Oxidative stress exacerbates mitochondrial and Endoplasmatic Reticulum (ER) dysfunction and produces subcellular remodelling and abnormalities of calcium handling and imbalance within the diabetic cardiomyocytes, which is characterized by calcium cytosolic overloading and reduced mitochondrial ATP production [61]. The $\mathrm{ER}$, through negative regulation of insulin's metabolic signalling, additionally impairs calcium homeostasis. There is a release of calcium from the ER into cytosol and reduced activity of the sarcoplasmatic reticulum calcium pump [62]. The consequences of these changes are modifications in the calcium sensitivity of regulatory proteins involved in the regulation of the cardiac actomyosin system, leading to impaired left ventricular function [62]. As an initial dysfunction, prolonged diastolic relaxation time, hour later on cardiomyocyte apoptosis due to the formation of mitochondrial permeability transition pore has been observed $[63,64]$.

\section{Renin-angiotensin-aldosterone and sympathetic system}

Hyperinsulinemia causes overactivation of the renin-angiotensinaldosterone system [65]. This leads to cardiac insulin resistance and the activation of mitogen activated protein kinases, which promote fibroblast proliferation while inducing cardiomyocyte fibrosis and apoptosis [66]. The serum level of aldosterone is increased in the prediabetic and diabetic condition and triggers LV hypertrophy, fibrosis and cardiac remodelling [67]. Both angiotensin II and aldosterone cause increased production of ROS and the activation of $\mathrm{NADPH}$ oxidase, and they therefore increase cytosolic oxidative stress [68]. Aldosterone also aggravates cardiac fibrosis by triggering proinflammatory factors through activation of matrix metalloproteinases and the transforming growth factor $\beta$ (TGF- $\beta$ ) [69]. There are reports of overactivation of the sympathetic system in the pre-diabetic and diabetic condition that further contributes to metabolic abnormalities. The association of blunted sympathetic responsiveness and insulin resistance, and disturbed sympathetic neurobiology is characterized by augmented resting sympathetic nervous activity and blunted sympathetic responsiveness to oral glucose ingestion [69].

\section{Electrophysiological}

Patients with diabetes mellitus are at higher risk of cardiac arrhythmias and sudden death. Autonomic neuropathy and/or cardiac repolarization abnormalities such as prolonged QT interval and altered T-waves of the diabetic heart also increases electrical instability. Diabetes is the strongest predictor of Atrial Fibrillation (AF) progression and that diabetic patients frequently have asymptomatic episodes of $\mathrm{AF}$ with silent arrhythmia progression and significantly alters the cardiac electrophysiology throughout several complex mechanisms greatly contributing to create an electrical instability of the heart, which may lead to potentially life-threatening arrhythmias and sudden cardiac death [70].

\section{Genetic}

Understanding the influences of genetic and environmental factors would provide insights into the pathomechanism of type 2 diabetes and cardiovascular diseases. In twin study, 14 different risk factors were simultaneously assessed in Monozygotic (MZ) and Dizygotic (DZ) adult twin pairs without diabetes and known cardiovascular diseases in order to determine the genetic and environmental influences on cardiometabolic risk factors [71].

\section{Advanced glycation end products (AGEs)}

Advanced glycation end products (AGEs) are proteins or lipids that become glycated after exposure to sugars. AGEs are prevalent in the diabetic vasculature and contribute to the development of atherosclerosis. The presence and accumulation of AGEs in many different cell types affect extracellular and intracellular structure and function. AGEs contribute to a variety of microvascular and macrovascular complications through the formation of cross-links between molecules in the basement membrane of the extracellular matrix and by engaging the receptor for advanced glycation end products (RAGE).

To conclude, according to current knowledge, DC is caused by different pathogenetic changes leading to diastolic and systolic dysfunction in diabetes complications with even more cardiovascular events. Further understanding of the mechanisms responsible for the onset of the functional and structural complications in the diabetic heart will undoubtedly aid the development of more precise therapeutics for the treatment of diabetic cardiomyopathy.

\section{References}

1. Emerging Risk Factors Collaboration, Seshasai SR, Kaptoge S, Thompson A Di Angelantonio E, et al. (2011) Diabetes mellitus, fasting glucose, and risk of cause-specific death. N Engl J Med 364: 829-841.

2. Hobbs FD (2006) Type-2 diabetes mellitus related cardiovascular risk: new options for interventions to reduce risk and treatment goals. Atheroscler Suppl 7: 29-32.

3. Shaw JE, Sicree RA, Zimmet PZ (2010) Global estimates of the prevalence of diabetes for 2010 and 2030. Diabetes Res Clin Pract 87: 4-14.

4. Nakano $\mathrm{Y}$, Tobe T, Choi Miura NH, Mazda T and Tomita M: Isolation and characterization of GBP28, a novel gelatin binding protein purified from human plasma. J Biochem 1996; 120: 80312. 
5. Gulati A, Jabbour A, Ismail TF, Guha K, Khwaja J, et al. (2013) Association of fibrosis with mortality and sudden cardiac death in patients with nonischemic dilated cardiomyopathy. JAMA 309: 896-908.

6. Falcão-Pires I, Leite-Moreira AF (2012) Diabetic cardiomyopathy: understanding the molecular and cellular basis to progress in diagnosis and treatment. Heart Fail Rev 17: 325-344.

7. Connelly KA, Kelly DJ, Zhang Y, Prior DL, Martin J, et al. (2007) Functional structural and molecular aspects of diastolic heart failure in the diabetic (mRen2)27 rat. Cardiovasc Res 76: 280-291

8. Connelly KA, Zhang YL, Advani A, Advani SL, Thai K, et al. (2013) DPP-4 inhibition attenuates cardiac dysfunction and adverse remodeling following myocardial infarction in rats with experimental diabetes. Cardiovasc Ther 2013; 31: 259-267.

9. Palmieri V, Capaldo B, Russo C, laccarino M, Pezzullo S, et al. (2008) Uncomplicated type 1 diabetes and preclinical left ventricular myocardial dysfunction: insights from echocardiography and exercise cardiac performance evaluation. Diabetes Res Clin Pract 79: 262-268.

10. Paulus WJ, Tschöpe C (2013) A novel paradigm for heart failure with preserved ejection fraction: comorbidities drive myocardial dysfunction and remodeling through coronary microvascular endothelial inflammation. J Am Coll Cardiol 62 263-271.

11. Wood P, Piran S, Liu PP (2011) Diastolic heart failure: progress, treatment challenges, and prevention. Can J Cardiol 27: 302-310.

12. Satpathy C, Mishra TK, Satpathy R, Satpathy HK, Barone E (2006) Diagnosis and management of diastolic dysfunction and heart failure. Am Fam Physician 73: $841-846$

13. Yu X, Tesiram YA, Towner RA, Abbott A, Patterson E, et al. (2007) Early myocardial dysfunction in streptozotocin-induced diabetic mice: a study using in vivo magnetic resonance imaging (MRI). Cardiovasc Diabetol 6: 6.

14. Fang ZY, Yuda S, Anderson V, Short L, Case C, et al. (2003) Echocardiographic detection of early diabetic myocardial disease. J Am Coll Cardiol 41: 611-617.

15. Rijzewijk LJ, van der Meer RW, Lamb HJ, de Jong HW, Lubberink M, et al. (2009) Altered myocardial substrate metabolism and decreased diastolic function in nonischemic human diabetic cardiomyopathy: studies with cardiac positron emission tomography and magnetic resonance imaging. J Am Coll Cardiol 54: 1524-1532.

16. Gilbert RE, Connelly K, Kelly DJ, Pollock CA, Krum H (2006) Heart failure and nephropathy: catastrophic and interrelated complications of diabetes. Clin J Am Soc Nephrol 1: 193-208.

17. Bajraktari G, Koltai MS, Ademaj F, Rexhepaj N, Qirko S, et al. (2006) Relationship between insulin resistance and left ventricular diastolic dysfunction in patients with impaired glucose tolerance and type 2 diabetes. Int J Cardiol 110: 206-211.

18. Huynh K, Kiriazis H, Du XJ, Love JE, Jandeleit-Dahm KA, et al. (2012) Coenzyme Q10 attenuates diastolic dysfunction, cardiomyocyte hypertrophy and cardiac fibrosis in the $\mathrm{db} / \mathrm{db}$ mouse model of type 2 diabetes. Diabetologia 55: 1544-1553.

19. Yu CM, Lin H, Yang H, Kong SL, Zhang Q, et al. (2002) Progression of systolic abnormalities in patients with "isolated" diastolic heart failure and diastolic dysfunction. Circulation 105: 1195-1201.

20. Vinereanu D, Nicolaides E, Tweddel AC, Mädler CF, Holst B, et al. (2003) Subclinical left ventricular dysfunction in asymptomatic patients with Type II diabetes mellitus, related to serum lipids and glycated haemoglobin. Clin Sci (Lond) 105: 591-599.

21. Bernardo BC, Weeks KL, Pretorius L, McMullen JR (2010) Molecular distinction between physiological and pathological cardiac hypertrophy: experimental findings and therapeutic strategies. Pharmacol Ther 128: 191-227.

22. Edgley AJ, Tare M, Evans RG, Skordilis C, Parkington HC (2008) In vivo regulation of endothelium-dependent vasodilation in the rat renal circulation and the effect of streptozotocin-induced diabetes. Am J Physiol Regul Integr Comp Physiol 295: R829-839.

23. Daniels A, van Bilsen M, Goldschmeding $R$, van der Vusse GJ, van Nieuwenhoven FA (2009) Connective tissue growth factor and cardiac fibrosis. Acta Physiol (Oxf) 195: 321-338.

24. Van Linthout S, Seeland U, Riad A, Eckhardt O, Hohl M, et al. (2008) Reduced MMP-2 activity contributes to cardiac fibrosis in experimental diabetic cardiomyopathy. Basic Res Cardiol 103: 319-327.
25. Huynh K, McMullen JR, Julius TL, Tan JW, Love JE, et al. (2010) Cardiacspecific IGF-1 receptor transgenic expression protects against cardiac fibrosis and diastolic dysfunction in a mouse model of diabetic cardiomyopathy. Diabetes 59: 1512-1520.

26. Ritchie R H, Love J E, Huynh K, Bernardo B C, Henstridge D C, et al. (2012) Enhanced phosphoinositide 3-kinase(p110a) activity prevents diabetes induced cardiomyopathy and superoxide generation in a mouse model of diabetes. Diabetologia 55: 3369-3381.

27. Ritchie RH, Leo CH, Qin C, Stephenson EJ, Bowden MA, et al. (2013) Low intrinsic exercise capacity in rats predisposes to age-dependent cardiac remodeling independent of macrovascular function. Am J Physiol Heart Circ Physiol 304: H729-739.

28. D'Souza A, Howarth FC, Yanni J, Dobryznski H, Boyett MR, et al. (2011) Left ventricle structural remodelling in the prediabetic Goto-Kakizaki rat. Exp Physiol 96: 875-888.

29. Yeshao W, Gu J, Peng X, Nairn AC, Nadler JL (2005) Elevated glucose activates protein synthesis in cultured cardiac myocytes. Metabolism 54: 1453-1460.

30. Lee SC, Pervaiz S (2007) Apoptosis in the pathophysiology of diabetes mellitus. Int J Biochem Cell Biol 39: 497-504

31. Fiordaliso F, Li B, Latini R, Sonnenblick EH, Anversa P, et al. (2000) Myocyte death in streptozotocin-induced diabetes in rats in angiotensin II- dependent Lab Invest 80: 513-527.

32. Cai L, Li W, Wang G, Guo L, Jiang Y, et al. (2002) Hyperglycemia-induced apoptosis in mouse myocardium: mitochondrial cytochrome C-mediated caspase-3 activation pathway. Diabetes 51: 1938-1948.

33. Yoon YS, Uchida S, Masuo O, Cejna M, Park JS, et al. (2005) Progressive attenuation of myocardial vascular endothelial growth factor expression is a seminal event in diabetic cardiomyopathy: restoration of microvascular homeostasis and recovery of cardiac function in diabetic cardiomyopathy after replenishment of local vascular endothelial growth factor. Circulation 111: 2073 2085.

34. Rifki OF, Hill JA (2012) Cardiac autophagy: good with the bad. J Cardiovasc Pharmacol 60: 248-252.

35. Yamaguchi O, Taneike M, Otsu K (2012) Cooperation between proteolytic systems in cardiomyocyte recycling. Cardiovasc Res 96: 46-52.

36. Oltman CL, Richou LL, Davidson EP, Coppey LJ, Lund DD, et al. (2006) Progression of coronary and mesenteric vascular dysfunction in Zucker obese and Zucker diabetic fatty rats. Am J Physiol Heart Circ Physiol 291: H1780 1787.

37. Woodman OL, Malakul W, Cao AH, Xu Q, Ritchie RH (2008) Atrial natriuretic peptide prevents diabetes-induced endothelial dysfunction. Life Sci 82: 847854

38. Yao D, Brownlee M (2010) Hyperglycemia-induced reactive oxygen species increase expression of the receptor for advanced glycation end products (RAGE) and RAGE ligands. Diabetes 59: 249-255.

39. Held C, Gerstein HC, Yusuf S, Zhao F, Hilbrich L, et al. (2007) Glucose levels predict hospitalization for congestive heart failure in patients at high cardiovascular risk. Circulation 115: 1371-1375.

40. Sánchez-Barriga JJ, Rangel A, Castañeda R, Flores D, Frati AC, et al. (2001) Left ventricular diastolic dysfunction secondary to hyperglycemia in patients with type II diabetes. Arch Med Res 32: 44-47.

41. Sun Z, Ernsberger P (2007) Marked insulin resistance in obese spontaneously hypertensive rat adipocytes is ameliorated by in vivo but not in vitro treatment with moxonidine. J Pharmacol Exp Ther 320: 845-852.

42. Reaven GM, Lithell H, Landsberg L (1996) Hypertension and associated metabolic abnormalities--the role of insulin resistance and the sympathoadrenal system. N Engl J Med 334: 374-381.

43. Robins SJ, Lyass A, Zachariah JP, Massaro JM, Vasan RS (2011) Insulin resistance and the relationship of a dyslipidemia to coronary heart disease: the Framingham Heart Study. Arterioscler Thromb Vasc Biol 31: 1208-1214.

44. How OJ, Aasum E, Severson DL, Chan WY, Essop MF, et al. (2006) Increased myocardial oxygen consumption reduces cardiac efficiency in diabetic mice. Diabetes 55: 466-473.

45. Gray S, Kim JK (2011) New insights into insulin resistance in the diabetic heart. Trends Endocrinol Metab 22: 394-403. 
Citation: Roever L, Casella-Filho A, Dourado PMM, Resende ES, Chagas ACP (2014) Cardiovascular Complications in Diabetes. J Diabetes Metab 5: 415 doi:10.4172/2155-6156.1000415

Page 6 of 6

46. Dhalla NS, Rangi S, Zieroth S, Xu YJ (2012) Alterations in sarcoplasmic reticulum and mitochondrial functions in diabetic cardiomyopathy. Exp Clin Cardiol 17: 115-120.

47. Dhalla NS, Takeda N, Rodriguez-Leyva D, Elimban V (2014) Mechanisms of subcellular remodeling in heart failure due to diabetes. Heart Fail Rev 19: 8799.

48. Galloway CA, Yoon Y (2013) Mitochondrial morphology in metabolic diseases. Antioxid Redox Signal 19: 415-430.

49. Rodrigues B, Cam MC, McNeill JH (1998) Metabolic disturbances in diabetic cardiomyopathy. Mol Cell Biochem 180: 53-57.

50. Wild S, Byrne CD (2004) The role of treatment to increase HDL-cholesterol and decrease triglyceride concentrations in prevention of coronary heart disease in Type 2 diabetes. Diabet Med 21 Suppl 4: 8-11.

51. Boden G (2003) Effects of free fatty acids (FFA) on glucose metabolism: significance for insulin resistance and type 2 diabetes. Exp Clin Endocrinol Diabetes 111: 121-124

52. Sun X, Pan H, Tan H, Yu Y (2012) High free fatty acids level related with cardiac dysfunction in obese rats. Diabetes Res Clin Pract 95: 251-259.

53. Greer JJ, Ware DP, Lefer DJ (2006) Myocardial infarction and heart failure in the $\mathrm{db} / \mathrm{db}$ diabetic mouse. Am J Physiol Heart Circ Physiol 290: H146-153.

54. van der Horst IC, de Boer RA, Hillege HL, Boomsma F, Voors AA, et al. (2010) Neurohormonal profile of patients with heart failure and diabetes. Neth Heart J 18: 190-196.

55. Forbes JM, Cooper ME (2013) Mechanisms of diabetic complications. Physio Rev 93: 137-188.

56. Ceriello A (2003) New insights on oxidative stress and diabetic complications may lead to a "causal" antioxidant therapy. Diabetes Care 26: 1589-1596.

57. van Campenhout $A$, van Campenhout CM, Lagrou AR, Manuel-y-Keenoy $B$ (2003) Transferrin modifications and lipid peroxidation: implications in diabetes mellitus. Free Radic Res 37: 1069-1077.

58. Feng Z, Hu W, Tang MS. Trans-4-hydroxy-2-nonenal inhibits nucleotide excision repair in human cells: a possible mechanism for lipid peroxidationinduced carcinogenesis. Proc Natl Acad Sci USA 2004, 101:8598-8602
59. Gray S, Kim JK (2011) New insights into insulin resistance in the diabetic heart Trends Endocrinol Metab 22: 394-403.

60. Minamino T, Komuro I, Kitakaze M (2010) Endoplasmic reticulum stress as a therapeutic target in cardiovascular disease. Circ Res 107: 1071-1082.

61. Abe T, Ohga Y, Tabayashi N, Kobayashi S, Sakata S, et al. (2002) Left ventricular diastolic dysfunction in type 2 diabetes mellitus model rats. Am J Physiol Heart Circ Physiol 282: H138-148.

62. Gorman AM, Healy SJ, Jäger R, Samali A (2012) Stress management at the ER: regulators of ER stress-induced apoptosis. Pharmacol Ther 134: 306-316.

63. Lastra G, Dhuper S, Johnson MS, Sowers JR (2010) Salt, aldosterone, and insulin resistance: impact on the cardiovascular system. Nat Rev Cardiol 7: 577-584.

64. Palomeque J, Delbridge L, Petroff MV (2009) Angiotensin II: a regulator of cardiomyocyte function and survival. Front Biosci (Landmark Ed) 14: 51185133.

65. Yoshimura M, Anzawa R, Mochizuki S (2008) Cardiac metabolism in diabetes mellitus. Curr Pharm Des 14: 2521-2526.

66. Manrique C, Lastra G, Habibi J, Wei Y, Morris EM, et al. (2007) Methods in the evaluation of cardiovascular renin angiotensin aldosterone activation and oxidative stress. Methods Mol Med 139: 163-179.

67. Catena C, Colussi G, Brosolo G, logna-Prat L, Sechi LA (2012) Aldosterone and aldosterone antagonists in cardiac disease: what is known, what is new. Am J Cardiovasc Dis 2: 50-57.

68. Straznicky NE, Grima MT, Sari Cl, Eikelis N, Lambert EA, et al. (2012) Neuroadrenergic dysfunction along the diabetes continuum: a comparative study in obese metabolic syndrome subjects. Diabetes 61: 2506-2516.

69. Pappone C1, Santinelli V (2010) Cardiac electrophysiology in diabetes. Minerva Cardioangiol 58: 269-276.

70. György Jermendy, Tamás Horváth, Levente Littvay, Rita Steinbach,Ádám $L$ Jermendy (2011) Effect of genetic and environmental influences on cardiometabolic risk factors: a twin study. Cardiovasc Diabetol 10: 96.

71. Goldin A, Beckman JA, Schmidt AM, Creager MA (2006) Advanced glycation end products: sparking the development of diabetic vascular injury. Circulation 114: 597-605 